\title{
Positron-electron correlation-polarization potentials for the calculation of positron collisions with atoms and molecules ${ }^{\star}$
}

\author{
Jan Franz ${ }^{\mathrm{a}}$ \\ Department of Theoretical Physics and Quantum Informatics, Faculty of Applied Physics and Mathematics, Gdansk University \\ of Technology, ul. Narutowicza 11/12, 80-233 Gdańsk, Poland
}

Received 23 September 2016 / Received in final form 23 December 2016

Published online 28 February 2017

(C) The Author(s) 2017. This article is published with open access at Springerlink.com

\begin{abstract}
We present correlation-polarization potentials for the calculation of scattering cross sections of positrons with atoms and molecules. The potentials are constructed from a short-range correlation term and a long-range polarization term. For the short-range correlation term we present four different potentials that are derived from multi-component density functionals. For the long-range polarization term we employ a multi-term expansion. Quantum scattering calculations are presented for low energy collisions of positrons with two atomic targets (argon and krypton) and two molecular targets (nitrogen and methane). For collision energies below the threshold for Positronium formation our calculations of scattering cross sections are in good agreement with recent data sets from experiments and theory.
\end{abstract}

\section{Introduction}

The understanding of positron scattering with atomic and molecular gases is important for the development of efficient positron cooling and trapping techniques [1]. Over the past ten years the experimental and calculated scattering cross sections for low-energy collisions of positrons with rare gas atoms, like argon and krypton, have converged within the experimental uncertainties [2-10]. For positron collisions with (polar and non-polar) molecules the situation is less favourable. The scattering from polar molecules with large dipole moments gives cross section with large peaks in forward direction. In measurements it is difficult to distinguish the unscattered particles from those scattered into a small cone in forward direction, which leads to the angular discrimination error [11-13]. Calculations for polar targets are difficult due to the slow convergence of the partial wave expansion. We reached good agreement between experiments and theory for targets such as water [14], pyrimidine [15] and tetrahydrofurane [16], after correcting the measurements for the angular discrimination error and the calculations by employing the Born approximation [17]. For non-polar molecules the angular discrimination is much smaller [3,18]. However, experimental cross sections for positron-molecule collisions are still rare, and show a much

\footnotetext{
* Contribution to the Topical Issue "Low-Energy Interactions related to Atmospheric and Extreme Conditions", edited by S. Ptasinska, M. Smialek-Telega, A. Milosavljevic and B. Sivaraman.

${ }^{a}$ e-mail: jfranz@mif.pg.gda.pl
}

larger spreading between different data sets. This is partly due to the weak positron beams used in older experiments. Calculations of the cross sections for positron collisions with non-polar molecules are considered more difficult than for atoms, because of the more complicated structure of the target. Even for small molecules, like hydrogen $\left(\mathrm{H}_{2}\right)$, there is still some disagreement between different theories and experiments (see for example Fedus et al. [19] for a comparison of the recent literature). Purely ab initio methods, like the multi-channel Schwinger method [20] and the R-matrix method [21], suffer from the difficulty in describing the correlation and long-range polarization interactions correctly. However, the accuracy of the R-matrix method can be improved systematically by using an additional pseudo-state basis set [22].

In this paper we present four correlation potentials to describe the short-range interations between a positron and the electrons of an isolated atom or molecule. The potentials are local and energy-independent. This makes them very useful for quantum scattering calculations and easy to implement into existing computer programs. For quantum scattering calculations we combine the shortrange correlation potential with a long-range polarization potential. Our derivation follows the recipe of Jain and Gianturco [23]. Jain and Gianturco are employing the multi-component density funtional of Boronski and Nieminen [24] for the short-range interaction and the $-\alpha / r^{4}$ potential for the long-range. This approach has been successfully used in many applications, for example for positron scattering from acetylene $[25,26]$ and tetrafluoromethane [27]. In this paper we want to show several 
alternatives to this approach. For the short-range correlation potentials, we want to suggest alternative functionals to the Boroński-Nieminen density functional [24]. These are the functionals by Boroński and Stachowiak [28] and by Drummond et al. [29,30]. For the long-range part of the polarization potential we include a number of terms of higher order. The importance of higher-order polarization terms in positron-atom scattering calculations has been shown previously by Gianturco and De Fazio [31], Gianturco et al. [32], and by Poveda et al. [33] .

In Section 2 we show derivations of the correlation potentials and give explicit equations for the short-range and long-range potentials. Furthermore we list all relevant numerical parameters and computational details about the calculations, which are presented in Section 3.

In Section 3 we present our calculations for elastic cross sections for positron collisions with the atoms argon and krypton and with the molecules nitrogen and methane. In Section 4 the paper ends with conclusions.

\section{Theoretical and computational methods}

\subsection{Hamiltonian}

For a given scattering channel $c$ the total Hamiltonian of the system

$$
H_{\text {total }}=H_{c}+V_{c}
$$

can be decomposed into the channel Hamiltonian $H_{c}$ and the interaction potential $V_{c}$ (see e.g. Chapter 14 in Joachain [34]). The channel Hamiltonian

$$
H_{c}=H_{\mathrm{mol}}+K_{e^{+}}
$$

is the sum of the Hamiltion $H_{\text {mol }}$ of the target molecule (or atom) and the kinetic energy of the impinging positron.

\subsection{Density functional theory for the positron-molecule interactions}

In the following we will work within the BornOppenheimer approximation and furthermore neglect couplings of the various nuclear and electronic spins. Within the framework of two-component density functional theory the total wavefunction

$$
\Psi_{\text {total }}\left(\mathbf{r}_{1}, \mathbf{r}_{2}, \ldots, \mathbf{r}_{\mathbf{N}} ; \mathbf{r}_{\mathbf{p}}\right)=\Phi_{\text {mol }}\left(\mathbf{r}_{1}, \mathbf{r}_{2}, \ldots, \mathbf{r}_{\mathbf{N}}\right) \times \varphi\left(\mathbf{r}_{\mathbf{p}}\right)
$$

can be expressed as a product of the electronic wavefunction of the target $\Phi_{\mathrm{mol}}\left(\mathbf{r}_{1}, \mathbf{r}_{2}, \ldots, \mathbf{r}_{\mathrm{N}}\right)$ and the positronic wavefunction $\varphi\left(\mathbf{r}_{\mathbf{p}}\right)$. The electronic wavefunction is the lowest-energy eigenfunction of the molecular Hamiltonian

$$
H_{\mathrm{mol}} \Phi_{\mathrm{mol}}\left(\mathbf{r}_{1}, \mathbf{r}_{2}, \ldots, \mathbf{r}_{\mathrm{N}}\right)=E_{\mathrm{GS}} \Phi_{\mathrm{mol}}\left(\mathbf{r}_{1}, \mathbf{r}_{2}, \ldots, \mathbf{r}_{\mathbf{N}}\right)
$$

where the eigenvalue $E_{\mathrm{GS}}$ is the energy of the electronic ground state.
With this ansatz the interaction potential $V_{c}$ can be written as

$$
V_{c}=V_{\mathrm{st}}+V_{\mathrm{pcp}}
$$

Here the static potential $V_{\text {st }}$ contains all electrostatic interactions between the positron and the target molecule, including the interactions with the nuclei and the electron charge cloud. The correlation-polarization potential $V_{\mathrm{pcp}}$ describes the correlation between the positron and the target electrons and the long-range polarization of the target. In the two-component density functional theory [35] this potential plays a similar role as the exchange-correlation potential in Kohn-Sham theory [36].

\subsection{Scattering equations}

Within the single-centre expansion (SCE) the positron wavefunction is expressed as [37]

$$
\varphi\left(\mathbf{r}_{\mathbf{p}}\right)=\sum_{l v} \frac{1}{r_{p}} u_{l v}\left(r_{\mathrm{p}}\right) X_{l v}\left(\hat{\mathbf{r}}_{\mathbf{p}}\right),
$$

where $r_{\mathrm{p}}$ is the radial distance of the positron from the scattering center and $\hat{\mathbf{r}}_{\mathbf{p}}$ is the unit vector in the direction of the positron. $X_{l v}$ are symmetry-adapted angular basis functions. The indices $l v$ represent the collection of indices, including the irreducible representation of the electronic state of the target molecule (or atom) and the angular momenta (for more details see e.g. Gianturco and Jain [37]). The functions $u_{l v}$ are the corresponding radial parts of the wavefunction. With this Ansatz for the positron wavefunction, we obtain, after integration over the electronic degrees of freedom, a set of coupled differential equations for the radial function of the positron

$$
\begin{aligned}
\left(\frac{d^{2}}{d r_{p}^{2}}-\frac{l(l+1)}{r_{p}^{2}}+k^{2}\right) & u_{l v}\left(r_{\mathrm{p}}\right) \\
& =2 \sum_{l^{\prime} v^{\prime}}\left\langle l v\left|\mathbf{V}_{\mathbf{c}}\right| l^{\prime} v^{\prime}\right\rangle u_{l^{\prime} v^{\prime}}\left(r_{\mathrm{p}}\right)
\end{aligned}
$$

where $k=\sqrt{2 E_{\mathrm{p}}}$ is related to the collision energy $E_{\mathrm{p}}$ of the positron in the usual way. The matrix elements of the coupling potential are given by

$$
\left\langle l v\left|\mathbf{V}_{\mathbf{c}}\right| l^{\prime} v^{\prime}\right\rangle=\int d \hat{\mathbf{r}}_{\mathbf{p}} X_{l v}^{*}\left(\hat{\mathbf{r}}_{\mathbf{p}}\right) V_{c}\left(\mathbf{r}_{\mathbf{p}}\right) X_{l^{\prime} v^{\prime}}\left(\hat{\mathbf{r}}_{\mathbf{p}}\right) .
$$

The set of coupled differential equations can be transformed into a set of Volterra integral equations (see e.g. Chap. 5 in Gianturco [38] and Franz [39])

$$
\begin{aligned}
\mathbf{U}(R)= & \int_{0}^{R}\{\mathbf{J}(R) \cdot \mathbf{N}(x) \\
& +\mathbf{N}(R) \cdot \mathbf{J}(x)\} \cdot \mathbf{V}(x) \cdot \mathbf{U}(x) d x
\end{aligned}
$$

Here $\mathbf{J}$ and $\mathbf{N}$ are diagonal matrices containing the spherical Ricatti-Bessel and Ricatti-Neumann functions, respectively. The matrix $\mathbf{U}(R)$ is related to the radial wave function. 
In the asymptotic region the $\mathbf{K}$-matrix is computed. From the $\mathbf{T}$-matrix, which is given by

$$
\mathbf{T}=1-(1-i \mathbf{K}) \cdot(1+i \mathbf{K})^{-1},
$$

the elastic integral cross section is computed as

$$
\sigma^{\text {elastic }}=\frac{\pi}{k^{2}} \sum_{l v} \sum_{l^{\prime} v^{\prime}}\left|T_{l^{\prime} v^{\prime}}^{l v}\right|^{2} .
$$

\subsection{Correlation-polarization potential}

The correlation-polarization potential is devided into a short-range and a long-range part [23]:

$$
V_{\mathrm{pcp}}\left(\mathbf{r}_{\mathrm{p}}\right)=\left\{\begin{array}{l}
V_{\text {corr }} \text { for } r_{\mathrm{p}} \leq r_{c} \\
V_{\mathrm{pol}} \text { for } r_{\mathrm{p}}>r_{c}
\end{array}\right.
$$

where $r_{c}$ is the outermost crossing point of the two potentials.

\subsubsection{Correlation potential}

We follow the ansatz of Gianturco and Jain [23] and derive the correlation potential as the functional derivative of the correlation energy

$$
V_{\text {corr }}=\gamma \frac{\delta}{\delta \rho_{-}} E_{\text {corr }}
$$

Here $\rho_{-}$is the electron density and $\gamma=\frac{1}{\sqrt{4 \pi}}$ is introduced for spherical avering. Jain and Gianturco are using the following relationship between the correlation energy $E_{\text {corr }}$ and the correlation enery $\epsilon_{\text {corr }}$ per particle and per unit volume

$$
E_{\mathrm{corr}}=\int d \mathbf{r} \rho_{-} \epsilon_{\mathrm{corr}} .
$$

This expression differs from the definition used originally by Boroński and Nieminen [24], Boroński and Stachowiak [28] and by Drummond et al. [29,30], by replacing the positron density by the electron density. The quantity $\epsilon_{\mathrm{corr}}$ is given as a function of the Wigner-Seitz radius

$$
r_{s}=\left(\frac{3}{4 \pi \rho_{-}}\right)^{-1}
$$

With this substitution we can express equation (13) as

$$
V_{\mathrm{corr}}=\gamma\left(1-\frac{1}{3} r_{s} \frac{d}{d r_{s}}\right) \epsilon_{\mathrm{corr}} .
$$

Various parametrizations for a positron in the homogenous electron gas are compared by Drummond et al. [29,30]. In this work we have implemented and tested four different parametrizations, which are described in the following paragraphs.

\subsubsection{Parametrization by Boroński and Nieminen}

Boroński and Nieminen [24] have constructed a functional in such a way that the high-density limit $\left(r_{s} \rightarrow 0\right)$ is given by the analytic expression of Arponen and Pajanne [40]. The low-density limit $\left(r_{s} \rightarrow \infty\right)$ is given by the energy of the Positronium negative ion $\left(\mathrm{Ps}^{-}\right)$. Between these two limiting cases interpolation formulae are used in such a way that the correlation energy is a continous and differentiable function of the electron density. Boroński and Nieminen [24] are giving the following parametrization for the correlation energy per particle:

- for $r_{s} \leq 0.302$

$$
\begin{aligned}
\epsilon_{\mathrm{corr}}^{\mathrm{BN} 86}= & \frac{1}{2}\left(-\frac{1.563}{\sqrt{r_{s}}}\right. \\
& \left.+\left(0.0507 \ln r_{s}-0.0807\right) \ln r_{s}+1.144\right)
\end{aligned}
$$

- for $0.302 \leq r_{s} \leq 0.56$

$$
\epsilon_{\mathrm{corr}}^{\mathrm{BN} 86}=\frac{1}{2}\left(-0.92305-\frac{0.05459}{r_{s}^{2}}\right)
$$

- for $0.56 \leq r_{s} \leq 8.0$

$$
\epsilon_{\mathrm{corr}}^{\mathrm{BN} 86}=\frac{1}{2}\left(-\frac{13.15111}{\left(r_{s}+2.5\right)^{2}}+\frac{2.8655}{\left(r_{s}+2.5\right)}-0.6298\right)
$$

- for the case $8.0 \leq r_{s} \leq \infty$

$$
\epsilon_{\mathrm{corr}}^{\mathrm{BN} 86}=\frac{1}{2}\left(-179856.2768 \rho_{-}^{2}+186.4207 \rho_{-}-0.524\right) .
$$

Gianturco and Jain [23] are giving the following explicit equations for the correlation potential:

- for $r_{s} \leq 0.302$

$$
\begin{aligned}
V_{\mathrm{corr}}^{\mathrm{BN} 86}= & \frac{\gamma}{2}\left(\frac{-1.82}{\sqrt{r_{s}}}\right. \\
& \left.+\left(0.051 \ln r_{s}-0.115\right) \ln r_{s}+1.167\right)
\end{aligned}
$$

- for $0.302 \leq r_{s} \leq 0.56$

$$
V_{\mathrm{corr}}^{\mathrm{BN} 86}=\frac{\gamma}{2}\left(-0.92305-\frac{0.09098}{r_{s}^{2}}\right)
$$

- for $0.56 \leq r_{s} \leq 8.0$

$$
\begin{aligned}
V_{\mathrm{corr}}^{\mathrm{BN} 86}= & \frac{\gamma}{2}\left(-\frac{8.7674 r_{s}}{\left(r_{s}+2.5\right)^{3}}\right. \\
& -\frac{-13.151+0.9552 r_{s}}{\left(r_{s}+2.5\right)^{2}} \\
& \left.-\frac{1.8655}{\left(r_{s}+2.5\right)}-0.6298\right)
\end{aligned}
$$

- for the case $8.0 \leq r_{s} \leq \infty$

$$
V_{\mathrm{corr}}^{\mathrm{BN} 86}=\frac{\gamma}{2}\left(-2 \times 179856.2768 \rho_{-}+186.4207\right) .
$$




\subsubsection{Parametrization by Boroński and Stachowiak}

Boroński and Stachowiak [28] have computed the correlation energy of one positron in the homogenous electron gas with the perturbed hypernetted chain (PHNC) approach over a broad range of electron densities. For various intervals Boroński and Stachowiak have interpolated the correlation energies and have connected the various density regimes so, that the energies and their derivatives with respect to the electron density are continous functions of the $r_{s}$ parameter. In the range $0.1<r_{s}<8$ the correlation energy is given by

$$
\epsilon_{\mathrm{corr}}^{\mathrm{PHNC}}=\frac{1}{2}\left(\frac{a}{\left(r_{s}-d\right)^{2}}+\frac{b}{\left(r_{s}-d\right)}+c\right) .
$$

Here we have an additional factor $\frac{1}{2}$ compared to equation (2) in Boroński and Stachowiak [28], because they are using Rydberg units (1 Rydberg $=\frac{1}{2}$ atomic units). Boroński and Stachowiak are providing two different parametrizations, which we will refer as $\mathrm{PHNCa}$ and $\mathrm{PH}-$ $\mathrm{NCb}$. The PHNCa functional is characterized by the following parameters:

for $0.1<r_{s}<1$

$$
\begin{aligned}
a & =0.033921278 \\
b & =-0.6968003 \\
c & =-0.3509695 \\
d & =-0.07
\end{aligned}
$$

and for $1<r_{s}<8$

$$
\begin{aligned}
a & =-2.385415 \\
b & =0.1732167 \\
c & =-0.4562613 \\
d & =-1.0
\end{aligned}
$$

The PHNCb functional is defined through the following parameters:

for $0.2<r_{s}<8$

$$
\begin{aligned}
a & =-1.428222 \\
b & =-0.1715698 \\
c & =-0.3927594 \\
d & =-0.67
\end{aligned}
$$

The corrlation potential is given by:

$$
V_{\mathrm{corr}}^{\mathrm{PHNC}}=\frac{\gamma}{2}\left(\frac{2 a d}{3\left(r_{s}-d\right)^{3}}+\frac{5 a+b d}{3\left(r_{s}-d\right)^{2}}+\frac{4 b}{3\left(r_{s}-d\right)}+c\right) .
$$

Here we are using both functionals also beyond the ranges given in the table. At high densities $\left(r_{s}<0.1\right.$ and $r_{s}<0.2$, respectively) the static potential dominates the interaction between the positron and the target, so that the exact form of the correlation potential does not matter too much. At low densities $\left(r_{s}>8\right)$ we are already in the regime of the asymptotic polarization potential, so that the exact form of the correlation potential in this density regime does not influence the cross section.

\subsubsection{Parametrization by Drummond et al.}

Drummond et al. $[29,30]$ are solving the problem of one positron in the homogenous electron gas by means of the Quantum Monte Carlo (QMC) method. For various electron densities the stabilization energy for the addition of one positron is calculated. Drummond et al. [29,30] are giving the following parametrization for the interpolation of the correlation energy

$$
\epsilon_{\mathrm{corr}}^{\mathrm{QMC}}=\frac{P}{Q},
$$

where

$$
P=A_{-1} r_{s}^{-1}+A_{0}+A_{1} r_{s}+C B_{2} r_{s}^{2}
$$

and

$$
Q=1+B_{1} r_{s}+B_{2} r_{s}^{2}
$$

Here

$$
C=-0.262005
$$

is the energy of the Positronium negative ion $\left(\mathrm{Ps}^{-}\right)$, which is the low-density limit $\left(r_{s} \rightarrow \infty\right)$. The paramenters are obtained by fitting to Quantum Monte Carlo calculations for various values of $r_{s}$ :

$$
\begin{aligned}
A_{-1} & =-0.260361 \\
A_{0} & =-0.261762 \\
A_{1} & =0.00375534 \\
B_{1} & =0.113718 \\
B_{2} & =0.0270912 .
\end{aligned}
$$

Comparing the parametrizations of Boroński and Stachowiak we can see that the PHNCa and PHNCb funtionals can be expressed in the parametric form suggested by Drummond et al.. The parameters are related in the following way:

$$
\begin{aligned}
A_{-1} & =0 \\
A_{0} & =\frac{a}{2 d^{2}}-\frac{b}{2 d}+\frac{c}{2} \\
A_{1} & =\frac{b}{2 d^{2}}-\frac{c}{d} \\
B_{1} & =-\frac{2}{d} \\
B_{2} & =\frac{1}{d^{2}} \\
C & =\frac{c}{2} .
\end{aligned}
$$

The correlation potential can be expressed as

$$
V_{\mathrm{corr}}^{\mathrm{QMC}}=\gamma\left(\frac{P}{Q}-\frac{r_{s}}{3}\left(\frac{P^{\prime} Q-P Q^{\prime}}{Q^{2}}\right)\right)
$$

where

$$
P^{\prime}=-A_{-1} r_{s}^{-2}+A_{1}+2 C B_{2} r_{s}
$$

and

$$
Q^{\prime}=B_{1}+2 B_{2} r_{s}
$$


Table 1. Parameters for the asymptotic potential. All values are in atomic units.

\begin{tabular}{ccccccc}
\hline Target & $\alpha_{\text {Dip }, 0}$ & $\alpha_{\text {Dip }, 2}$ & $\alpha_{\text {Quad }}$ & $\alpha_{\text {Oct }}$ & $B$ & $\gamma$ \\
\hline $\mathrm{Ar}$ & $11.070^{a}$ & - & $52.4^{b}$ & $490^{b}$ & $-164.3^{b}$ & $1083^{b}$ \\
$\mathrm{Kr}$ & $17.075^{a}$ & - & $92.7^{b}$ & $793^{b}$ & $-343.2^{b}$ & $2255^{b}$ \\
$\mathrm{~N}_{2}$ & $11.74^{d}$ & $4.593^{d}$ & $83.26^{e}$ & - & $-151.53^{g}$ & $927^{e}$ \\
$\mathrm{CH}_{4}$ & $16.39^{f}$ & - & $120.90^{f}$ & - & $-317.79^{g}$ & $2312^{f}$ \\
\hline
\end{tabular}

a Experimental values from Holm and Kerl [41,42] as cited in Schwerdtfeger [43]. ${ }^{b}$ From tabulated values in Gianturco et al. [32]. ${ }^{c}$ From explicitly correlated calculations by Bishop et al. [44-46]. ${ }^{d}$ Obtained from dipole oscillator strength distribution by Kumar and Meath [47]. ${ }^{e}$ From $\operatorname{CCSD}(\mathrm{T})$ calculations by Maroulis [48]. ${ }^{f}$ From $\operatorname{CCSD}(\mathrm{T})$ calculations by Maroulis [49]. ${ }^{g}$ From CCSD calculations by Coriani et al. [50].

\subsubsection{Polarization potential}

In the calculations on positron-atom scattering (here argon and krypton) we are using the asymptotic form suggested by Gianturco et al. [32]

$$
V_{\mathrm{pol}}\left(\mathbf{r}_{\mathrm{p}}\right)=-\frac{\alpha_{\text {Dip }, 0}}{2 r_{\mathrm{p}}^{4}}-\frac{\alpha_{\mathrm{Quad}}}{2 r_{\mathrm{p}}^{6}}-\frac{\alpha_{\text {Oct }}}{2 r_{\mathrm{p}}^{8}}+\frac{B}{2 r_{\mathrm{p}}^{7}}-\frac{\gamma}{24 r_{\mathrm{p}}^{8}},
$$

where $\alpha_{\text {Dip,0 }}$ is the isotropic static dipole polarizability, $\alpha_{\text {Quad }}$ is the isotropic static quadrupole polarizability, and $\alpha_{\text {Oct }}$ is the isotropic static octupole polarizability. $B$ is the dipole-dipole - isotropic quadrupole hyperpolarizability and $\gamma$ is the dipole-dipole - isotropic dipole-dipole hyperpolarizability. In the calculations on positron-molecule scattering (here nitrogen and methane) we are using the following form for the polarization potential

$$
\begin{aligned}
V_{\text {pol }}\left(\mathbf{r}_{\mathrm{p}}\right)= & -\frac{\alpha_{\text {Dip }, 0}}{2 r_{\mathrm{p}}^{4}}-\frac{\alpha_{\operatorname{Dip}, 2}}{2 r_{\mathrm{p}}^{4}} P_{2}\left(\cos \theta_{\mathrm{p}}\right) \\
& -\frac{\alpha_{\mathrm{Quad}}}{2 r_{\mathrm{p}}^{6}}+\frac{B}{2 r_{\mathrm{p}}^{7}}-\frac{\gamma}{24 r_{\mathrm{p}}^{8}},
\end{aligned}
$$

where $\alpha_{\mathrm{Dip}, 2}$ is the anisotropic static dipole polarizability and $P_{2}\left(\cos \theta_{\mathrm{p}}\right)$ is a Legendre polynomial. The values of the parameters which we are using in our calculations are collected in Table 1.

\subsection{Computational details}

\subsubsection{Electronic structure calculations for atoms}

We are using the MOLCAS program package [51] for the electron structure calculations of the atoms Ar and $\mathrm{Kr}$. The complete active space self-consistent-field (CASSCF) method is employed for calculating the electronic ground state wavefunctions and densities. For Ar the active space is generated by 8 active electrons in the orbitals $(3 s, 3 p, 3 d, 4 s, 4 p, 5 s, 5 p)$, which results in 64331 determinants. For $\mathrm{Kr}$ the active space is generated by 8 active electrons in the orbitals $(4 s, 4 p, 4 d, 5 s, 5 p, 6 s, 6 p)$, which results in 415306 determinants. We are using the contracted gaussian basis set for Douglas-Kroll-Heß calculations of triple zeta valence quality plus polarization functions (TZP-DKH) of Jorge et al. [52], which we obtained from the EMSL Basis Set Library [53,54]. The DouglasKroll-Heß method $[55,56]$ is used to account for scalar relativistic effects of the target wave function. In order to interface the CASSCF electron-density into our scattering code, we are using natural orbitals for the electronic ground state (here the ${ }^{1} \mathrm{~S}_{0}$ state). In natural orbitals the one-electron density matrix can be written as

$$
\left.\rho_{-}\left(\mathbf{x}_{1} ; \mathbf{x}_{1}^{\prime}\right)\right)=\sum_{i} n_{i} \bar{\psi}_{i}\left(\mathbf{x}_{1}\right) \bar{\psi}_{i}^{*}\left(\mathbf{x}_{1}^{\prime}\right)
$$

where the $\bar{\psi}_{i}$ are ortho-normal spin-orbitals with the population $n_{i}$. For more details see e.g. Chapters 5 and 8 in McWeeny [57] or Chapter 3 in Davidson [58].

\subsubsection{Electronic structure calculations for molecules}

We are using the TURBOMOLE program package [59] for the electronic structure calculations of the target molecules employing the B3LYP (Becke [60], threeparameter, Lee-Yang-Parr [61]) exchange-correlation functional.

For the nitrogen molecule $\left(\mathrm{N}_{2}\right)$ the calculations are done with a bondlength of $1.094 \AA$ and using the triplezeta basis set $6-311++\mathrm{G}^{* *}$ of Krihnan et al. [62] with polarization and diffuse functions.

For the methane molecule $\left(\mathrm{CH}_{4}\right)$ calculations are done at a CH-bondlength of $1.089 \AA$ using the TZP basis set of Barbieri et al. [63], which we augmented by diffuse functions of Dunning and Hay [64].

\subsubsection{Quantum scattering calculations}

We are using a modified version of the VOLPOS program package [65] for the single-centre-expansion of the electron densities, for the construction of the potential and the solution of the scattering equations by Volterra integration. In all calculations the radial grid was extended up to a distance of 146 bohr. All potentials are expanded up to $L_{\max }=24$ and the positronic wavefunction up to $l_{\max }=12$. The calculations for the atoms and the nitrogen molecule are done in the symmetry group $D_{2 h}$ and for methane in the $\mathrm{C}_{2 \mathrm{v}}$ group. All irreducible representations are included in the final cross sections. At the outermost radial grid point we generate the $\mathbf{K}$-matrices and compute the elastic integral cross section.

\section{Results and discussion}

In Figures 1 and 2 we compare our calculations for the Ar atom with cross sections from recent experiments and calculations. Figure 2 shows results from the measurements by Karwasz et al. [3], Jones et al. [7], and Zecca et al. [6]. The experimental data of Karwasz et al. [3] and Zecca et al. [6] does not contain corrections for angular discrimination. The data by Jones et al. [7] includes some angular correction and presents the total cross sections 


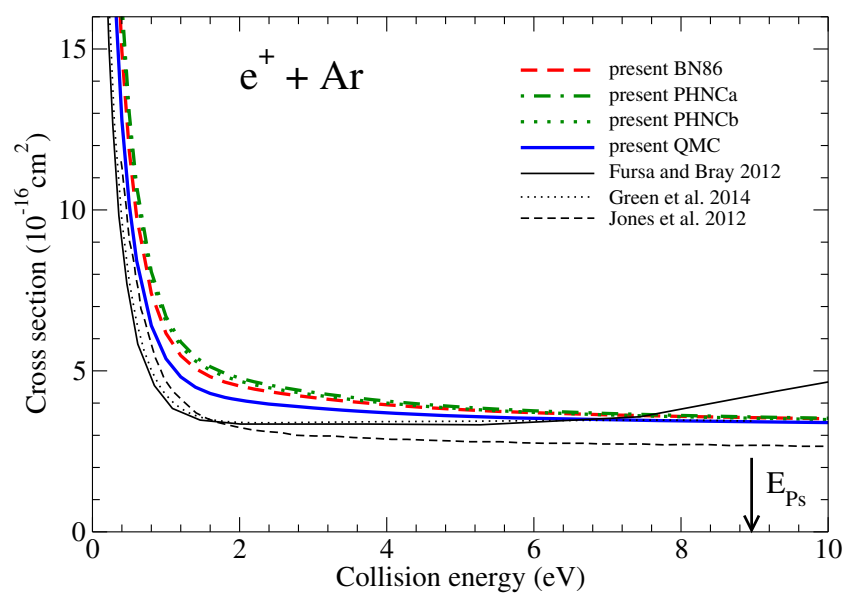

Fig. 1. Scattering cross section for Ar. Present calculations of elastic cross sections with the potentials BN86 (red dashed line), PHNCa (green dashed-dotted line), $\mathrm{PHNCb}$ (green dotted line), and QMC (blue solid line). Calculations by other groups: total cross sections from Fursa and Bray [5] (thin black solid line), elastic cross sections from Green et al. [4] (thin black dashed line), and Jones et al. [7] (thin black dotted line). For more details see main text.

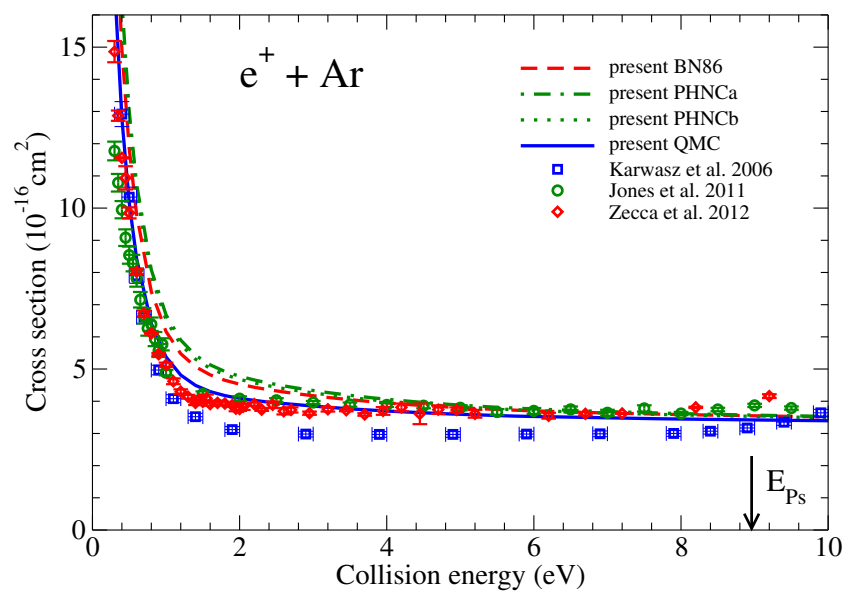

Fig. 2. Scattering cross section for Ar. Present calculations of elastic cross sections with the potentials BN86 (red dashed line), PHNCa (green dashed-dotted line), PHNCb (green dotted line), and QMC (blue solid line). Experimental total cross sections from Karwasz et al. [3] (blue squares) and Zecca et al. [6] (red diamonds). Total cross sections excluding Positronium formation from Jones et al. [7] (green circles), For more details see main text.

without Positronium formation. Figure 1 shows the calculations done by Fursa and Bray [5] using the convergentclose-coupling (CCC) method, by Green et al. [4] using many-body perturbation theory (MBPT), and by Jones et al. [7] using the relativistic optical potential (ROP) method. Our calculations and those by Green et al. [4] and Jones et al. [7] are done for elastic cross sections. The calculations by Fursa and Bray [5] and the experiments by Karwasz et al. [3] and Zecca et al. [6] are showing total cross sections. In the energy region above the threshold for Positronium formation and below $10 \mathrm{eV}$ the difference

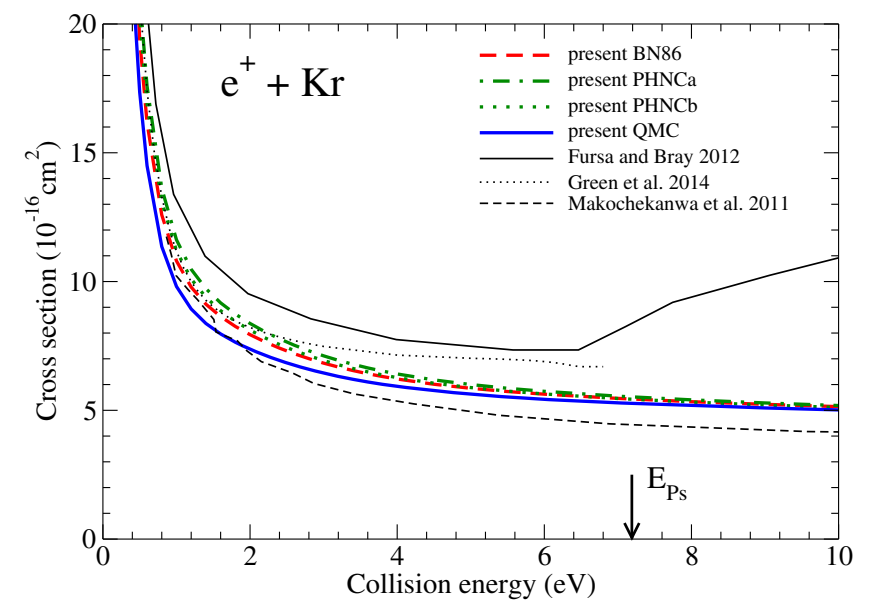

Fig. 3. Scattering cross section for Kr. Present calculations of elastic cross sections with the potentials BN86 (red dashed line), $\mathrm{PHNCa}$ (green dashed-dotted line), PHNCb (green dotted line), and QMC (blue solid line). Calculations by other groups: total cross sections from Fursa and Bray [5] (thin black solid line), elastic cross sections from Green et al. [4] (thin black dashed line), and Makochekanwa et al. [9] (thin black dotted line). For more details see main text.

between elastic and total cross section is mainly due to the channel for Positronium formation. This channel opens at

$$
E_{\mathrm{Ps}}=E_{\text {ion }}-E_{0}
$$

where $E_{\text {ion }}$ is the first ionization energy and $E_{0}=6.8 \mathrm{eV}$ is the binding energy of Ps. The first ionization energy of the ground state of the Ar-atom is given as $E_{\text {ion }}=$ $15.759610 \mathrm{eV}$ by Sansonetti and Martin in the Handbook of Basic Atomic Spectroscopic Data of the NIST database [66] based on experiments by Velchev et al. [67]. This places the threshold for Ps formation at $E_{\mathrm{Ps}}=$ $8.96 \mathrm{eV}$. This position is marked by an arrow in Figures 1 and 2 .

Our four sets of calculations give very similar results to each other. The potentials $\mathrm{PHNCa}$ and $\mathrm{PHNCb}$ are giving nearly identical cross sections. Both are slightly larger than those obtained with the BN86 potential. The QMC potential gives slighlty smaller cross sections than the other three potentials. This ordering holds over the complete energy range. The results from all four sets are within the experimental uncertainties of the three shown sets of experimental data. For collision energies below $5 \mathrm{eV}$ the calculations by Fursa and Bray [5], Green et al. [4] and Jones et al. [7] give smaller cross sections than those obtained in our calculations. For energies above $5 \mathrm{eV}$ and up to Ps-formation threshold the agreement between our calculations and those by Fursa and Bray [5] and by Green et al. [4] is better.

In Figures 3 and 4 we are showing a comparison of our four sets of computed cross sections for the Kr atom with a selection of recent calculated and experimental cross sections from the literature. Figure 4 shows the experimental data sets of Jay and Coleman [10], Makochekanwa et al. [9] and Zecca et al. [8]. Figure 3 shows the calculations done 


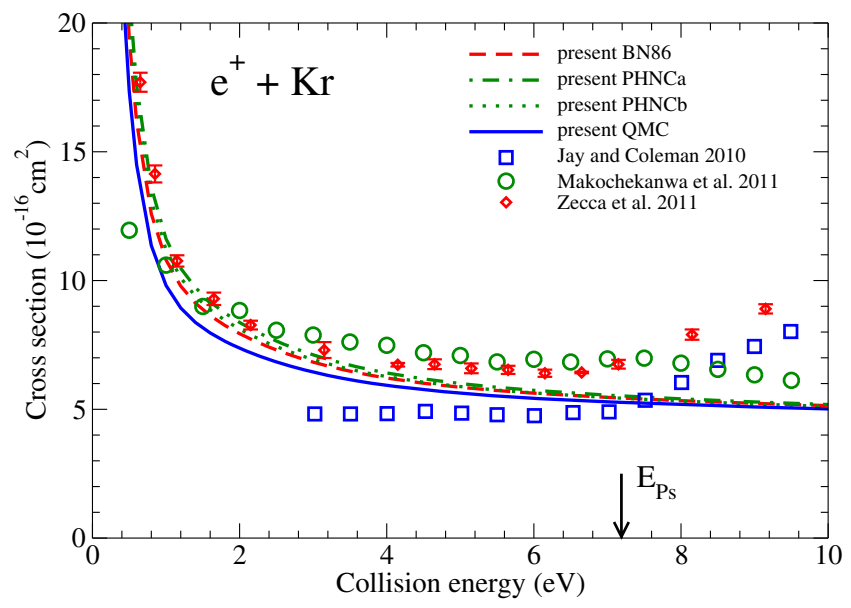

Fig. 4. Scattering cross section for Kr. Present calculations of elastic cross sections with the potentials BN86 (red dashed line), PHNCa (green dashed-dotted line), PHNCb (green dotted line), and QMC (blue solid line). Experimental total cross sections from Jay and Coleman [10] (blue squares) and Zecca et al. [8] (red diamonds). Total cross sections excluding Positronium formation from Makochekanwa et al. [9] (green circles). For more details see main text.

by Fursa and Bray [5] using the CCC-method, by Green et al. [4] using MBPT, and by Makochekanwa et al. [9] using the ROP method.

As for argon, our calculated cross sections are elastic cross sections and do not contain the Ps-formation channel. The same applies for the calculations by Green et al. [4] and Makochekanwa et al. [9]. The experimental data sets from Jay and Coleman [10] from Zecca et al. [8] and the calculations by Fursa and Bray [5] are total cross sections and contain the Ps-formation channel. The data by Jay and Coleman [10] are not absolute cross sections but are scaled to previous experiments. The experimental data from Makochekanwa et al. [9] present total cross section without Positronium formation. The first ionization energy of $\mathrm{Kr}$ in the electronic ground state is $13.99961 \mathrm{eV}$ as given in the NIST database [66] based on the measurements by Yoon and Glab [68] and by Brandi et al. [69]. This places the threshold for Ps formation at $7.19 \mathrm{eV}$ (see arrow in Figs. 3 and 4).

The cross sections from our four sets of calculations are very close to each other. Similar as for argon, the potentials $\mathrm{PHNCa}$ and $\mathrm{PHNCb}$ give nearly identical results and produce the largest cross sections of our models. The BN86 potential give slightly lower cross sections, followed by the QMC potential. The total cross sections from the experiments by Jay and Coleman [10] and by Zecca et al. [8] increase markedly at the threshold for Ps-formation. The CCC-method of Fursa and Bray [5] is reproducing the increase, while all other computations do not account for Ps-formation. Up to the Ps-formation threshold our calculated cross sections are slightly below the measured data of Makochekanwa et al. [9] and Zecca et al. [8] and slightly higher than the data by Jay and Coleman [10]. The CCC-calculations by Fursa and Bray [5] are

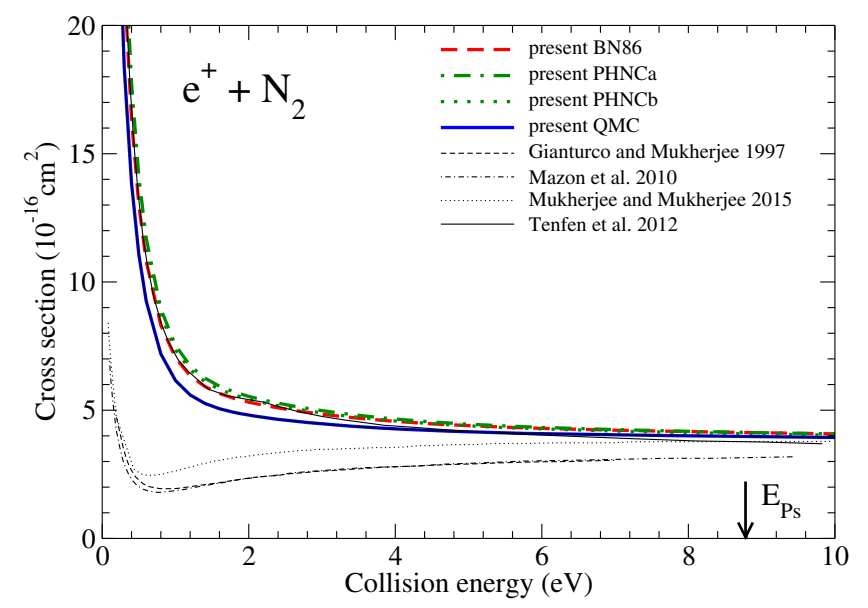

Fig. 5. Scattering cross section for $\mathrm{N}_{2}$. Present calculations of elastic cross sections with the potentials BN86 (red dashed line), PHNCa (green dashed-dotted line), PHNCb (green dotted line), and QMC (blue solid line). Calculations by other groups: vibrational close-coupling calculations from Gianturco and Mukherjee [74] (thin black dashed line), elastic cross sections from Mazon et al. [76] (thin black dashed-dotted line), ro-vibrational close-coupling calculations from Mukherjee and Mukherjee [75] (thin black dottd line), and elastic cross sections from Tenfen et al. [77] (thin black solid line). For more details see main text.

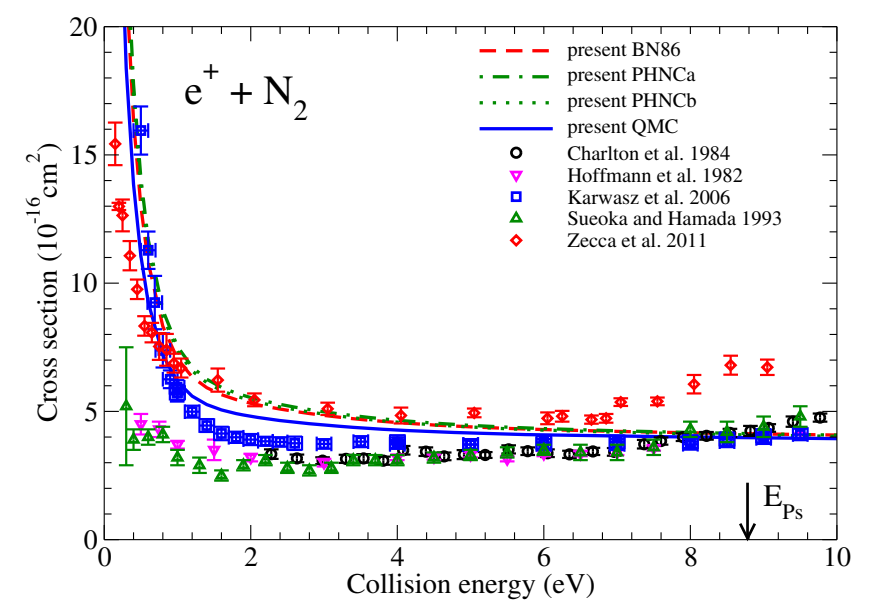

Fig. 6. Scattering cross section for $\mathrm{N}_{2}$. Present calculations of elastic cross sections with the potentials BN86 (red dashed line), PHNCa (green dashed-dotted line), PHNCb (green dotted line), and QMC (blue solid line). Experimental total cross sections from Charlton et al. [70] (black circles), Hoffmann et al. [71] (magenta down-triangles), Karwasz et al. [3] (blue squares), Sueoka and Hamada [72] (green up-triangles), and Zecca et al. [73] (red diamonds). For more details see main text.

giving the largest cross sections, followed by the MBPT calculations by Green et al. [4], which are slighly larger than our values. The ROP-calculations by Makochekanwa et al. [9] are larger than our values for collision energies below $2 \mathrm{eV}$ and smaller at higher energies.

Figures 5 and 6 show our calculated cross sections for the nitrogen molecule $\left(\mathrm{N}_{2}\right)$ using the four different 
potentials along with selected computed and measured cross sections from the literature. The measured cross sections in Figure 6 are from experiments by Charlton et al. [70] from 1984, Hoffmann et al. [71] from 1982, Sueoka and Hamada [72] from 1993, Karwasz et al. [3] from 2006, and Zecca et al. [73] from 2011. The shown experimental data does not contain any corrections for the angular discrimination error. Figure 5 also shows a selection of calculations from other groups: Gianturco and Mukherjee [74], Mukherjee and Mukherjee [75] Mazon et al. [76], and Tenfen et al. [77]. Our calculations are giving elastic cross sections using the fixed nuclei approximation in the body fixed reference frame. Therefore rotational and vibrational effects are neglected and no Ps-formation is included. The same is true for the calculated cross sections by Mazon et al. [76] and by Tenfen et al. [77]. The experimental data sets are showing total cross sections, which includes rotational, vibrational and Ps-formation channels. The calculated cross sections from Gianturco and Mukherjee [74] are including vibrational inelastic channels and those by Mukherjee and Mukherjee [75] are including vibrational and rotational inelastic channels. For $\mathrm{N}_{2}$ the first ionization energy of the ground state is at $15.581 \pm 0.008 \mathrm{eV}$ as measured by Trickl et al. [78]. This places the threshold for Ps-formation at about $8.78 \mathrm{eV}$ (see arrow in Figs. 5 and 6).

Below collision energies of $2 \mathrm{eV}$ the more recent experiments by Karwasz et al. [3] and Zecca et al. [73] show a large increase with decreasing energies, whereas the older measurements by Hoffmann et al. [71] and Sueoka and Hamada [72] stay more or less constant. Charlton et al. [70] do not provide data for these energies. Above collision energies of $2 \mathrm{eV}$ all five experiments give total cross sections similar within $1 \times 10^{-16} \mathrm{~cm}^{2}$. The experimental data sets show the increase in the total cross sections can be seen above the Ps-formation threshold. The cross sections obtained from our computations show the same ordering as observed for the other targets: the potentials PHNCa and PHNCb potentials are giving the largest cross sections, followed by the BN86 potential and the QMC potential. Our four calculations agree well with the more recent experiments by Karwasz et al. [3] and by Zecca et al. [73]. The calculated cross sections from Mukherjee and Gianturco [74] and from Mukherjee and Mukherjee [75] are too low when compared to recent experiments. The results from Gianturco and Mukherjee [74] are very close to those by Mazon et al. [76]. Slighly higher are the results from Mukherjee and Mukherjee [75]. The calculations by Tenfen et al. [77] are employing an ab initio potential to describe correlation and polarization. They are very close to our calculations using the BN86 potential. At low collision energies all calculations from the literature experience a minimum in the cross sections, which also seems to be in the older experimental data from Hoffman et al. [71] and Sueoka and Hamada [72], but not in the more recent experimental data from Karwasz et al. [3] and Zecca et al. [73].

In Figures 7 and 8 we show our results for positron collisions with methane $\left(\mathrm{CH}_{4}\right)$ along with some selected

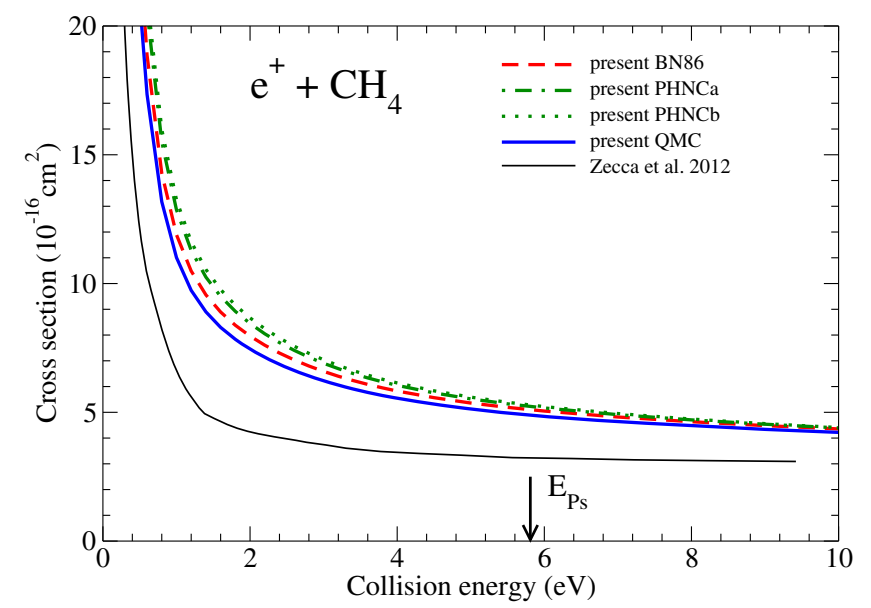

Fig. 7. Scattering cross section for $\mathrm{CH}_{4}$. Present calculations of elastic cross sections with the potentials BN86 (red dashed line), $\mathrm{PHNCa}$ (green dashed-dotted line), PHNCb (green dotted line), and QMC (blue solid line). Calculations of elastic cross sections by Zecca et al. [83] (thin black solid line). For more details see main text.

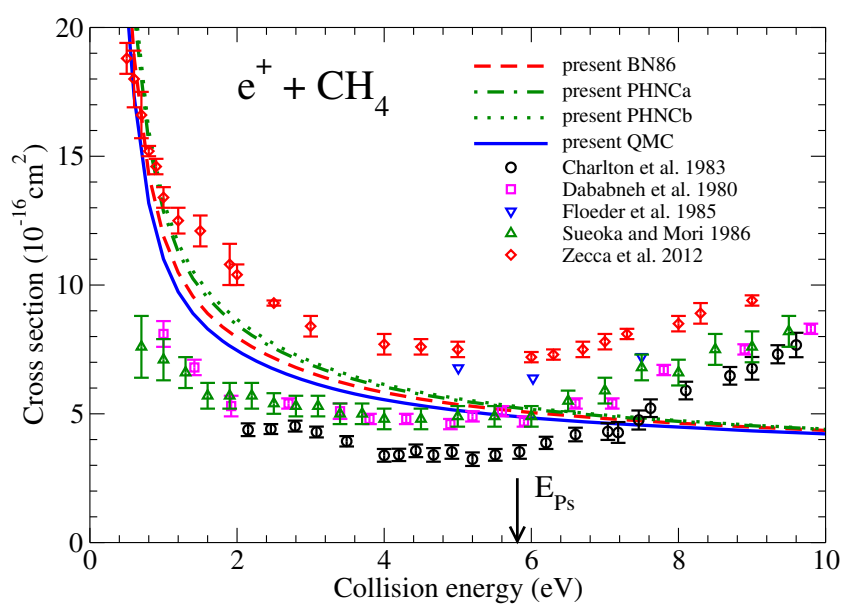

Fig. 8. Scattering cross section for $\mathrm{CH}_{4}$. Present calculations of elastic cross sections with the potentials BN86 (red dashed line), PHNCa (green dashed-dotted line), PHNCb (green dotted line), and QMC (blue solid line). Experimental total cross sections from Charlton et al. [79] (black circles), Dababneh et al. [80] (magenta squares), Floeder et al. [81] (blue downtriangles), Sueoka and Mori [82] (green up-triangles), and Zecca et al. [83] (red diamonds). For more details see main text.

theoretical and experimental results from the literature. The measured cross section data are from the experiments by Charlton et al. [79], Dababneh et al. [80], Floeder et al. [81], Sueoka and Mori [82], and Zecca et al. [83]. Also shown in Figure 7 are calculations done by Zecca et al. [83] using the Schwinger-method. Our calculated cross sections are elastic cross sections within the fixed-nuclei approximation in the body fixed reference frame. Therefore no rotational, vibrational and Ps-formation channels 
are included. The same applies to the calculations by Zecca et al. [83]. The experimental data sets are showing total cross sections and include all these channels. In methane the first ionization energy of the ground state is $12.61 \pm 0.4 \mathrm{eV}$ according to measurements by Berkowitz et al. [84]. This places the threshold for Ps-formation at $5.81 \mathrm{eV}$ (see arrow in Figs. 7 and 8).

Our four sets of calculations are very close to each other. As for the other targets, the PHNCa and PHNCb potentials gives the largest cross sections, followed by the BN86 potential and the QMC potential. The largest measured cross sections are those by Zecca et al. [83] and Floeder et al. [81]. The smallest cross sections have been measured by Charlton et al. [79]. Our calculated cross sections are inbetween the various experimental data sets. All experimental data sets show an increase in the cross total section around the Ps-formation threshold. This feature is not reproduced by our calculations, because we do not include this channel. The calculated cross sections from Zecca et al. [83] are much lower than our calculated cross sections and are close to the measured cross sections by Charlton et al. [79].

In order to estimate the effect of neglecting the vibrational inelastic channels, one can have a look at the computations done by Nishimura and Gianturco [85] for vibrational inelastic cross sections. Summing over all four vibrational modes, the vibrational inelastic cross section appears to be $0.3 \times 10^{-16} \mathrm{~cm}^{2}$ at the lowest vibrational threshold $(0.2 \mathrm{eV})$, reaches a maximum of roughly $0.8 \times 10^{-16} \mathrm{~cm}^{2}$ at $0.5 \mathrm{eV}$ and decreases realatively quickly. At $5.0 \mathrm{eV}$ the sum of the vibrational inelastic cross sections is around $0.2 \times 10^{-16} \mathrm{~cm}^{2}$. The inclusion of the vibrational inelastic channels will bring our cross sections closer to the measured values of Zecca et al. [83] and Floeder et al. [81], but cannot fully explain the differences between our calculations the their measurements.

\section{Conclusions}

In this paper we present four local positron-electron correlation potentials. All four potentials are derived from existing multicomponent density functionals. The BN86-potentials is derived from the Boroński-Nieminen functional. The QMC-potential is based on QuantumMonte-Carlo calculations by Drummond et al. [29,30]. The potentials PHNCa and $\mathrm{PHNCb}$ are based on perturbed hypernetted chain calculations by Boroński and Stachowiak [28]. All four correlation potentials are used to describe short-range interactions between positrons and electrons and are combined with a multi-term expansion to describe the long-range polarization forces between a positron and a multi-electron atom or molecule. For all potentials we present simple analytical forms, which allows an easy implementation into existing computer programs.

The potentials are applied in quantum scattering calculations for elastic collisions of positrons with the two rare gas atoms argon and krypton and the molecules nitrogen and methane. The elastic cross sections are compared with scattering data from recent experiments and calculations. The elastic cross sections computed with the PHNCa-potential and PHNCb-potential are giving very similar results to each other. For all targets and all collision energies they are are larger than the cross sections obtained with the BN86-potential. The QMC-potential gives the smallest cross sections in this set. For argon and nitrogen all four potentials give cross sections that are in good agreement with the most recent experiments and calculations. For krypton the present calculations are between the various data sets from the most recent experiments and calculations. For methane our calculated cross sections are lower than the most recent experimental data sets and larger than older data sets or other calculations from the literature.

To conclude, we recommend the QMC potential for future calculations of cross sections, because the mathematical expression is more simple and it is valid over the whole electron-density regime.

This work is supported by the grant 2014/15/D/ST2/02358 of the Narodowe Centrum Nauki (National Science Center Poland). The author thanks and Prof. Edward Boroński (Opole University) and Prof. Grzegorz Karwasz (Nicolaus Copernicus University, Toruń) for helpful discussions. The author acknowledges computational resources made availabe by Prof. Sigrid Peyerimhoff at the Mulliken Center for Theoretical Chemistry (University of Bonn).

\section{References}

1. J.R. Danielson, D.H.E. Dubin, R.G. Greaves, C.M. Surko, Rev. Mod. Phys. 87, 247 (2015)

2. J. Franz, K. Fedus, G.P. Karwasz, Eur. Phys. J. D 70, 155 (2016)

3. G.P. Karwasz, D. Pliszka, R.S. Brusa, Nucl. Instrum. Methods B 247, 68 (2006)

4. D.G. Green, J.A. Ludlow, G.F. Gribakin, Phys. Rev. A 90, $032712(2014)$

5. D.V. Fursa, I. Bray, New J. Phys. 14, 035002 (2012)

6. A. Zecca, L. Chiari, E. Trainotti, D.V. Fursa, I. Bray, A. Sarkar, S. Chattopadhyay, K. Ratnavelu, M.J. Brunger, J. Phys. B 45, 015203 (2012)

7. A.C.L. Jones, C. Makochekanwa, P. Caradonna, D.S. Slaughter, J.R. Machachek, R.P. McEachran, J.P. Sullivan, S.J. Buckman, A.D. Stauffer, I. Bray, D.V. Fursa, Phys. Rev. A 83, 032701 (2011)

8. A. Zecca, L. Chiari, E. Trainotti, D.V. Fursa, I. Bray, M.J. Brunger, Eur. Phys. J. D 64, 317 (2011)

9. C. Makochekanwa, J.R. Machacek, A.C.L. Jones, P. Caradonna, D.S. Slaughter, R.P. McEachran, J.P. Sullivan, S.J. Buckman, S. Bellm, B. Lohmann, D.V. Fursa, I. Bray, D.W. Mueller, A.D. Stauffer, M. Hoshino, Phys. Rev. A 83, 032721 (2011)

10. P.M. Jay, P.G. Coleman, Phys. Rev. A 82, 012701 (2010)

11. J.P. Sullivan, C. Makochekanwa, A. Jones, P. Caradonna, D.S. Slaughter, J. Machacek, R.P. McEachran, D.W. Mueller, S.J. Buckman, J. Phys. B 44, 035201 (2011) 
12. W.E. Kauppila, T.S. Stein, J.H. Smart, M.S. Dababneh, Y.K. Ho, J.P. Downing, V. Pol, Phys. Rev. A 24, 725 (1981)

13. C.K. Kwan, W.E. Kauppila, R.A. Lukaszew, S.P. Parikh, T.S. Stein, Y.J. Wan, M.S. Dababneh, Phys. Rev. A 44, 1620 (1991)

14. K.L. Baluja, R. Zhang, J. Franz, J. Tennyson, J. Phys. B 403515 (2007)

15. J. Franz, F.A. Gianturco, Phys. Rev. A 88, 042711 (2013)

16. J. Franz, F.A. Gianturco, J. Chem. Phys. 139, 204309 (2013)

17. N. Sanna, F.A. Gianturco, Comput. Phys. Commum. 114, $142(1998)$

18. G.P. Karwasz, A. Karbowski, Z. Idziaszek, R.S. Brusa, Nucl. Instrum. Methods B 266, 471 (2008)

19. K. Fedus, J. Franz, G.P. Karwasz, Phys. Rev. A 91, 062701 (2015)

20. R.C. Carvalho, M.T. do N. Varella, M.A.P. Lima, E.P. Silva, J.S.E. Germano, Nucl. Instrum. Methods Phys. Res. Sect. B 171, 33 (2000)

21. J. Franz, K.L. Baluja, R. Zhang, J. Tennyson, Nucl. Instrum. Methods B 266419 (2008)

22. R. Zhang, K.L. Baluja, J. Franz, J. Tennyson, J. Phys. B 44035203 (2011)

23. A. Jain, F.A. Gianturco, J. Phys. B 24, 2387 (1991)

24. E. Boroński, R.M. Nieminen, Phys. Rev. B 34, 3820 (1986)

25. J. Franz, F.A. Gianturco, Eur. Phys. J. D 39, 407 (2006)

26. J. Franz, F.A. Gianturco, K.L. Baluja, J. Tennyson, R. Carey, R. Montuoro, R.R. Lucchese, T. Stoecklin, P. Nicholas, T.L. Gibson, Nucl. Instrum. Methods B 266, 425 (2008)

27. J. Franz, I. Baccarelli, S. Caprasecca, F.A. Gianturco, Phys. Rev. A 80012709 (2009)

28. E. Boroński, H. Stachowiak, Phys. Rev. B 57, 6215 (1998)

29. N.D. Drummond, P. López Ríos, C.J. Pickard, R.J. Needs, Phys. Rev. B 82, 035107 (2010)

30. N.D. Drummond, P. López Ríos, C.J. Pickard, R.J. Needs, Phys. Rev. Lett. 107, 207402 (2011)

31. F.A. Gianturco, D. De Fazio, Phys. Rev. A 50, 4819 (1994)

32. F.A. Gianturco, A. Jain, J.A. Rodriguez-Ruiz, Phys. Rev. A 48, 4321 (1993)

33. L.A. Poveda, A. Dutra, J.R. Mohallem, D. Assafrao, Phys. Rev. A 87, 052702 (2013)

34. C.J. Joachain, Quantum Collision Theory (North Holland Publishing, Amsterdam, 1975)

35. R.M. Nieminen, E. Boroński, L.J. Lantto, Phys. Rev. B 32, 1377 (1985)

36. W. Kohn, L.J. Sham, Phys. Rev. 140, A1133 (1965)

37. F.A. Gianturco, A. Jain, Phys. Rep. 143, 347 (1986)

38. F.A. Gianturco, The Transfer of Molecular Energies by Collision: Recent Quantum Treatments (Springer-Verlag, Berlin, 1979)

39. J. Franz, J. Math. Phys. 56, 012104 (2015)

40. J. Arponen, E. Pajanne, Ann. Phys. 121, 343 (1979)

41. U. Hohm, K. Kerl, Mol. Phys. 69, 803 (1990)

42. U. Hohm, K. Kerl, Mol. Phys. 69, 819 (1990)

43. P. Schwerdtfeger, Atomic Static Dipole Polarizabilities, in Computational Aspects of Electric Polarizability Calculations: Atoms, Molecules and Clusters, edited by G. Maroulis (IOS Press, Amsterdam, 2006), pp. 1-32, with the addition: Updated static dipole polarizabilities are available as pdf file from the CTCP website at Massey University: http://ctcp.massey.ac.nz/ dipole-polarizabilities
44. D.M. Bishop, J. Pipin, S.M. Cybulski, Phys. Rev. A 43, 4845 (1991)

45. D.M. Bishop, S. Lam, J. Chem. Phys. 89, 1571 (1988)

46. D.M. Bishop, J. Pipin, Int. J. Quantum Chem. 45, 349 (1993)

47. A. Kumar, W.J. Meath, Theor. Chim. Acta 82, 131 (1992)

48. G. Maroulis, J. Chem. Phys. 118, 2673 (2003)

49. G. Maroulis, Chem. Phys. Lett. 226, 420 (1994)

50. S. Coriani, C. Hättig, P. Jorgensen, A. Rizzo, K. Ruud, J. Chem. Phys. 109, 7176 (1998)

51. MOLCAS Version 5.4, K. Andersson, M. Barysz, A. Bernhardsson, M.R.A. Blomberg, Y. Carissan, D.L. Cooper, M.P. Fülscher, L. Gagliardi, C. de Graaf, B.A. Heß, G. Karlström, R. Lindh, P.-Å. Malmqvist, T. Nakajima, P. Neogrády, J. Olsen, B.O. Roos, B. Schimmelpfennig, M. Schütz, L. Seijo, L. SerranoAndrés, P.E.M. Siegbahn, J. Stålring, T. Thorsteinsson, V. Veryazov, P.-O. Widmark, Lund University, Sweden (2002)

52. F.E. Jorge, A. Canal Neto, G.G. Camiletti, S.F. Machado, J. Chem. Phys. 130, 064108 (2009)

53. D. Feller, J. Comp. Chem. 17, 1571 (1996)

54. K.L. Schuchardt, B.T. Didier, T. Elsethagen, L. Sun, V. Gurumoorthi, J. Chase, J. Li, T.L. Windus, J. Chem. Inf. Model. 47, 1045 (2007)

55. M. Douglas, N.M. Kroll, Ann. Phys. 82, 89 (1974)

56. G. Jansen, B.A. Heß, Phys. Rev. A 93, 6016 (1993)

57. R. McWeeny, Methods of Molecular Quantum Mechanics (Academic Press, London, 1996)

58. E.R. Davidson, Reduced Density Matrices in Quantum Chemistry (Academic Press, New York, 1976)

59. Turbomole version 5-6, Copyright (C) 2002 University of Karlsruhe

60. A.D. Becke, J. Chem. Phys. 98, 5648 (1993)

61. C. Lee, W. Yang, R.G. Parr, Phys. Rev. B 37, 785 (1988)

62. R. Krishnan, J.S. Binkley, R. Seeger, J.A. Pople, J. Chem. Phys. 72, 650 (1980)

63. P.L. Barbieri, P.A. Fantin, F.E. Jorge, Mol. Phys. 104, 2945 (2006)

64. T.H. Dunning Jr., P.J. Hay, in Modern Theoretical Chemistry, edited by H.F. Schaefer III (Plenum Press, New York, 1976), Vol. 3, pp. 1-28

65. R. Curik, F.A. Gianturco, N. Sanna, J. Phys. B 33, 2705 (2000)

66. J.E. Sansonetti, W.C. Martin, J. Chem. Phys. Ref. Data 341559 (2005)

67. I. Velchev, W. Hogervorst, W. Ubachs, J. Phys. B 32, L511 (1999)

68. S. Yoon, W.L. Glab, J. Phys. B 27, 1433 (1994)

69. F. Brandi, W. Hogervorst, W. Ubacks, J. Phys. B 35, 1071 (2002)

70. M. Charlton, G. Laricchia, T.C. Griffith, G.L. Wright, G.R. Heyland, J. Phys. B 17, 4945 (1984)

71. K.R. Hoffman, M.S. Dababneh, Y.F. Hsieh, W.E. Kauppila, V. Pol, J.H. Smart, T.S. Stein, Phys. Rev. A 25, 1393 (1982)

72. O. Sueoka, A. Hamada, J. Phys. Soc. Jpn 62, 2669 (1993)

73. A. Zecca, L. Chiari, A. Sarkar, M.J. Brunger, New J. Phys. 13, 115001 (2011)

74. F.A. Gianturco, T. Mukherjee, Phys. Rev. A 55, 1044 (1997)

75. T. Mukherjee, M. Mukherjee, Phys. Rev. A 91, 062706 (2015) 
76. K.T. Mazon, W. Tenfen, S. Michelin, F. Arretche, M.-T. Lee, M.M. Fujimoto, Phys. Rev. A 82, 032704 (2010)

77. W. Tenfen, K.T. Mazon, S.E. Michelin, F. Arretche, Phys. Rev. A 86, 042706 (2012)

78. T. Trickl, E.F. Cromwell, Y.T. Lee, A.H. Kung, J. Chem. Phys. 91, 6006 (1989)

79. M. Charlton, T.C. Griffith, G.R. Heyland, G.L. Wright, J. Phys. B 16, 323 (1983)

80. M.S. Dababneh, Y.F. Hsieh, W.E. Kauppila, C.K. Kwan, S.J. Smith, T.S. Stein, M.N. Uddin, Phys. Rev. A 38, 1207 (1988)

81. K. Floeder, D. Fromme, W. Raith, A. Schwab, G. Sinapius, J. Phys. B 18, 3347 (1985)

82. O. Sueoka, S. Mori, J. Phys. B 19, 4035 (1986)
83. A. Zecca, L. Chiari, E. Trainotti, A. Sarkar, S. d'A. Sanchez, M.H.F. Bettega, M.T. do N. Varella, M.A.P. Lima, M.J. Brunger, Phys. Rev. A 85, 012707 (2012)

84. J. Berkowitz, J.P. Greene, H. Cho, B. Ruscic, J. Chem. Phys. 86, 674 (1987)

85. T. Nishimura, F.A. Gianturco, Phys. Rev. A 65, 062703 (2002)

Open Access This is an open access article distributed under the terms of the Creative Commons Attribution License (http://creativecommons.org/licenses/by/4.0), which permits unrestricted use, distribution, and reproduction in any medium, provided the original work is properly cited. 